\title{
Bona fide Stochastic Resonance: A view point from stochastic
}

\author{
energetics. \\ Debasis Dan* and A. M. Jayannavar \\ Institute of Physics, Sachivalaya Marg, Bhubaneswar 751005, India.
}

(Dated: May 24, 2022)

\begin{abstract}
We investigate the resonance type behaviour of an overdamped Brownian particle in a bistable potential driven by external periodic signal. It has been shown previously that the input energy pumped into the system by the external drive shows resonance type behaviour as function of noise strength. We further extend this idea to study the behaviour as function of frequency of the external driving force and show the occurrence of similar nonmonotonic behaviour, which can be ascribed as a signature of bona fide stochastic resonance. Both weak and strong driving limit has been explored indicating the occurrence of marginal supra-threshold stochastic resonance in a bistable potential system.
\end{abstract}

PACS numbers: 05.40.-a, 02.50.Ey

*Electronic address: dan@iopb.res.in 


\section{INTRODUCTION}

Stochastic Resonance (SR) is a nonlinear phenomena where the combined effect of noise and nonlinearity (bistable systems or threshold systems) leads to an enhanced response of a weak periodic signal with addition of noise of optimal intensity. This counter intuitive phenomena has been observed experimentally and in numerical and analog simulations [1]. However, there has been a lot of discussions in recent years regarding the validity of SR as bona fide stochastic resonance 2]. The archetype of SR models is represented by a simple symmetric bistable potential driven by a zero mean Gaussian white noise and an external sinusoidal bias. The response of such system has been mainly characterized by the response amplitude (RA) of the periodic component of the process $\langle x(t)>$ or the signal to noise ratio (SNR). Both these response functions show typical resonance type feature with increasing noise amplitude $D$ [1]. This resonance has been attributed to the matching of escape rate across the barrier and the frequency of the external periodic drive. An obvious conclusion is the occurrence of a similar peak with increasing frequency of the bias. But such nonmonotonic behaviour has not been observed in spite of exhaustive numerical and analytical studies on SNR and RA. However, ref. [3] shows the existence of peak in SNR with increasing frequency for a system with rectangular potential barrier and for a special type of driving.

Other criteria have been proposed to study the resonance behaviour as function of frequency, thus characterizing SR as a bona fide resonance. Gammaitoni et. al. have shown that residence time distribution $\mathrm{N}(\mathrm{T})$ has a resonant behaviour as function of forcing frequency. Marchesoni et. al. recently showed numerically that in a Schmitt trigger $\mathrm{N}(\mathrm{T})$ shows a peak with frequency for both weakly and strongly driven system. The relationship 
between SR and synchronization of passages from one well to another can also be characterized by hysteresis loop area [4, 5]. This loss can be taken as a measure of SR and SR is shown as a bona fide resonance. Recently it is argued that input energy is also a good measure of SR 7]. This energy is equivalent to the work done by the external agent which drives the potential periodically. The input energy not only shows peaking behaviour with temperature (noise strength) but also takes into account only the interwell behaviour. In the conventional SNR both intrawell as well as interwell motion is taken into account and hence for small driving frequency and noise strength the motion is predominantly dominated by the intrawell oscillations citeiwai. Hence the peak in the input energy is a better indicator of matching of escape rate and the external driving frequency. This assertion has been made by taking into account the detailed comparison between various measures of SR.

In this work we show that input energy not only correctly shows the matching condition for noise induced escape rate and the external periodic drive, but resonance type behaviour is also obtained as function of $\omega$ (frequency of external drive), a signature of Bonafide SR. Bonafide SR is a relatively new term in stochastic dynamics, by which one means the actual matching of the time scales $T_{e s}$ (barrier escape time) and $T_{\omega}$ (period of external drive, $2 \pi / \omega$ ). This is manifested as a peak in the plot of input energy with $T_{\omega}$ and $D$. The existence of SR in supra-threshold regime has also been demonstrated. We make an exhaustive study throughout the parameter regime of our problem and show the detailed behaviour of SR peak as function of $D, \omega$ and $A$ in our model.

\section{MODEL}

We consider an overdamped Brownian particle moving in a bistable potential $V(x, t)=$ $-x^{2} / 2+x^{4} / 4-A x \sin (\omega t)$ under the influence of a zero mean white Gaussian noise $\xi(t)$ 
with correlations $<\xi(t) \xi\left(t^{\prime}\right)>=2 D \delta\left(t-t^{\prime}\right)$. The Langevin equation for such system is

$$
\dot{x}(t)=-V^{\prime}(x, t)+\xi(t)
$$

and the corresponding Fokker Planck equation (FPE) is

$$
\frac{\partial P(x, t)}{\partial t}=-\frac{\partial}{\partial x}\left(V^{\prime}(x, t)-D \frac{\partial}{\partial x}\right) P(x, t)
$$

where $P(x, t)$ is the probability density of the particle at position $x$ at time $t$. The barrier height $\Delta V=0.25$. We consider both weak forcing $A x_{m}<\Delta V$ as well as strong forcing $A x_{m}>\Delta V$ limit. Depending on the parameter regime, the Brownian particle is either dominated by intra well or inter well oscillations. For small driving force the motion is mostly dominated by intrawell oscillations. At certain optimal noise strength the interwell motion is enhanced due to the combined effect of noise and weak periodic modulation. Such resonant enhancement of interwell motion is termed as Stochastic Resonance (SR). In this particular case the oscillatory driving force keeps the system away from equilibrium. The energy required to drive the system $\left(E_{i n}\right)$ can be calculated from Sekimoto's stochastic energetics formalism [6] and its has been shown that $E_{\text {in }}$ depends nonmonotonically on the noise strength $D$. Since the mean current in the system is zero, hence no work is done by the system and all the input energy is dissipated into the bath. This suggests that input energy is related to dissipative loss or hysteresis loss in the system [4, 5]. Our effort is to find the signatures of SR in the input energy of the system as function of system parameters $\omega$ and $D$.

The input energy per period $\left(T_{\omega}\right)$ is defined as $[6,[7]$

$$
E_{\text {in }}=\int_{t_{0}}^{t_{0}+T_{\omega}}<\frac{\partial V(x, t)}{\partial t} d t>=-A \omega \int_{t_{0}}^{t_{0}+T_{\omega}}<x(t) \cos (\omega t) d t>.
$$

The average $\langle.$.$\rangle is done over an ensemble of particles. The asymptotic probability$ 
distribution $P(x, t)$ is calculated by solving the FPE (2). The input energy $E_{\text {in }}$ can be rewritten in terms of asymptotic distribution as

$$
E_{i n}=\int_{-\infty}^{\infty} d x \int_{t_{0}}^{t_{0}+T_{\omega}} d t x(t) \cos (\omega t) P_{a s y}(x, t) .
$$

where $P_{a s y}(x, t)=P_{a s y}\left(x, t+T_{\omega}\right)$. This distribution is obtained after the initial transients have died down and asymptotically probability density assumes a unique limiting periodic distribution in time. The maxima of $E_{i n}$ as function of $D$ is taken as a signature of SR. We numerically solve eqn. (21) by method of finite difference to obtain the asymptotic distribution $P_{a s y}(x, t) . E_{\text {in }}$ is obtained by numerically integrating eqn. (41). Throughout this work all the physical quantities are in dimensionless unit [1].

\section{RESULTS AND DISCUSSIONS}

The input energy shows a rich structure as function of $A, \omega$ and $D$. We mainly concentrate on low amplitude drive, $A x_{m} / \Delta<1$. In this regime input energy shows a sharp maxima both as function of $D$ and $\omega$. In fig. 1 we plot $E_{i n}$ as function of $D$ for $A=0.1$ and different values of $\omega$. The peak in the input energy can be attributed to the synchronization of escape from the potential well and the external periodic drive as has been extensively discussed in previous literatures [7]. With increasing $\omega$ the temperature at which $E_{\text {in }}$ peaks increases as shown in fig. 1. The most favourable condition for hopping to other potential well is when the barrier height is minimum. When $\omega$ is increased the Brownian particle spends less time in the most favourable condition and hence stronger fluctuations are needed to cross the barrier. Barrier height also decreases with increase in amplitude of external drive. Hence for reasons similar to above the resonance peak shift towards lower 
temperature as shown in the inset, where we have plotted $E_{\text {in }}$ vs $D$ for various values of $A$ as mentioned in the caption. However, our main motive is to check whether these resonance features are also observed when the frequency of the external drive is varied. In fig. 2 we plot $E_{i n}$ as function of $\omega$ for $A=0.1$ and different values of temperature. Unlike other response function like SNR, RA which characterize SR and has monotonic dependence on $\omega$ , input energy shows a nonmonotonic and a peaking behaviour with $\omega$. For small amplitude drive and at low temperature the resonant frequency is very close to half the Kramer's rate for the unperturbed system ( the resonance condition being $1 / r_{k}=T_{\omega} / 2 \rightarrow \omega / \pi=r_{k}$ ). For $D=0.2$, the Kramer's rate $\left(r_{k}=\frac{1}{\sqrt{2} \pi} \exp (-0.25 / D)\right)$ is 0.0645 , which is very close to $\omega / \pi=0.14 / \pi=0.0446$. With higher amplitude the resonance peak shifts to higher frequency for a given temperature as shown in the inset. Thus SR as a bona fide resonance is established.

It is known that for $A>>A_{\max }$, where $A_{\max }$ is the dynamical threshold above which deterministic switch events take place driven by the periodic signal alone (absence of noise), SR is not observed. The dynamical threshold depends on both the modulation frequency and the wave form $A(t)$. For the form given in eqn.( (1) $A_{\max } / A_{t h}=1+\beta(\Omega / a)$, where $\beta=(2 \sqrt{3} / \pi) g_{1}$ and $g_{1}$ is the smallest zero of the Airy function $A i^{\prime}(-x)$, is order of unity 8]. We investigate the marginal supra-threshold regime $\left(A>A_{\max }\right)$ and show that $\mathrm{SR}$ is observed with increasing temperature provided $A$ is not very large compared to $A_{\max }$. The occurrence of SR in this regime is related to noise induced stability [5, 8]. In fig. 3] we plot input energy for four different valued of $A$ at $\omega=1.0$. Figure 4 shows the corresponding deterministic trajectories. As shown in fig. 4. $A=0.85$ is the threshold, i.e, the particle is just able to cross the barrier at $x=0$. Hence higher values of $A$ is above dynamic threshold. For $A>0.85$ the input energy shows a resonance peak with increasing temperature. This 
peak in $E_{\text {in }}$ in supra-threshold regime is shown for a very small window of $A$. For the specific parameter values as above, the peak vanishes for $A>0.91$. This peak can be ascribed to resonant trapping as discussed in previous literature [5, 8]. However, in supra-threshold regime $E_{\text {in }}$ clearly exhibits peaking behaviour as function of $\omega[9]$ for all values of $A$. This is more akin to conventional resonance (absence of barrier).

In conclusion we have calculated that the input energy pumped into the system by an external drive using the method of stochastic energetics. This input energy is shown to be a good quantitative measure of SR. Moreover SR is shown to be a bona fide resonance.

[1] L. Gammaitoni, P. Hanggi, P. Jung, and F. Marchesoni, Rev. Mod. Phys. 70, 223 (1998).

[2] L. Gammaitoni, F. Marchesoni, and S. Santucci, Phys. Rev. Lett. 74, 1052 (1995); M. H. Choi, R. F. Fox, and P. Jung, Phys. Rev. E 57, 6335 (1998); G. Giacomelli, F. Marin, and I. Rabbiosi, Phys. Rev. Lett. 82, 675 (1999); F. Marchesoni, L. Gammaitoni, F. Apostolico, and S. Santucci, Phys. Rev. E 62, 146 (2000).

[3] V. Berdichevsky amd M. Gitterman, J. Phys. A 29, L447 (1996).

[4] M. C. Mahato and S. R. Shenoy, Phys. Rev. E 50, 2503 (1994); M. C. Mahato and A. M. Jayannavar, Phys. Rev. E 55, 6266 (1997).

[5] M. C. Mahato and A. M. Jayannavar, Mod. Phys. Lett. B 11, 815 (1997); M. C. Mahato and A. M. Jayannavar, Physica A 248, 138 (1998).

[6] K. Sekimoto, J. Phys. Soc. Jpn. 66, 6335 (1997); F. Takagi and T. Hondou, Phys. Rev. E 60, 4954 (1999).

[7] T. Iwai, Physica A 300, 350 (2001); T. Iwai, J. Phys. Soc. Jpn. 70, 353 (2001).

[8] F. Apostolico, L. Gammaitoni, F. Marchesoni, and S. Santucci, Phys. Rev. E, 55, 36 (1997); 
R. N. Mantegna and B. Spagnolo, Phys. Rev. Lett 76, 563 (1996).

[9] A. L. Pankratov, Phys. Rev. E 65, 022101 (2002). 


\section{FIGURE CAPTIONS .}

Fig 1. $E_{\text {in }}$ vs $D$ for $A=0.1$. The inset shows the plot of $E_{\text {in }}$ vs $D$ for $\omega=0.1,0.2,0.3$ from top to bottom at $A=0.2$.

Fig. 2. $E_{\text {in }}$ vs $\omega$ for $A=0.1$. Inset is the plot of $E_{\text {in }}$ vs $\omega$ at $A=0.2$ for $D=0.15,0.2,0.25$ from top to bottom curve respectively.

Fig. 3. $E_{\text {in }}$ vs $D$ at $\omega=1.0$ for large amplitude $A=0.85,0.87,0.90$ and 0.92 respectively.

Fig. 4. The determistic trajectories $(D=0)$ for amplitudes $A=0.85,0.90$ and 0.92 . All these trajectories cross the barrier at $x=0$. 


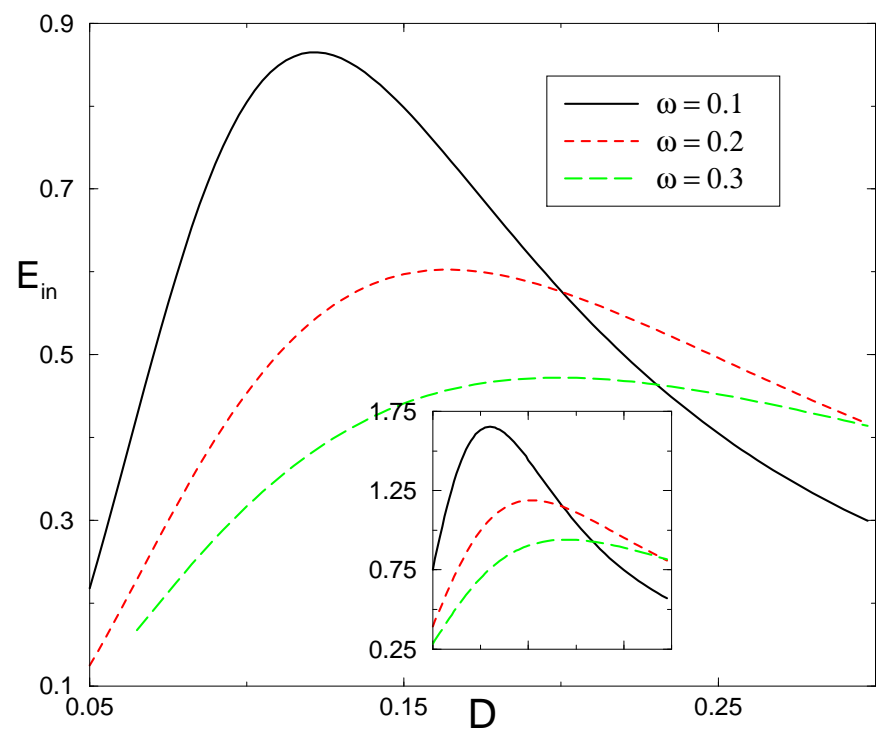

FIG. 1:

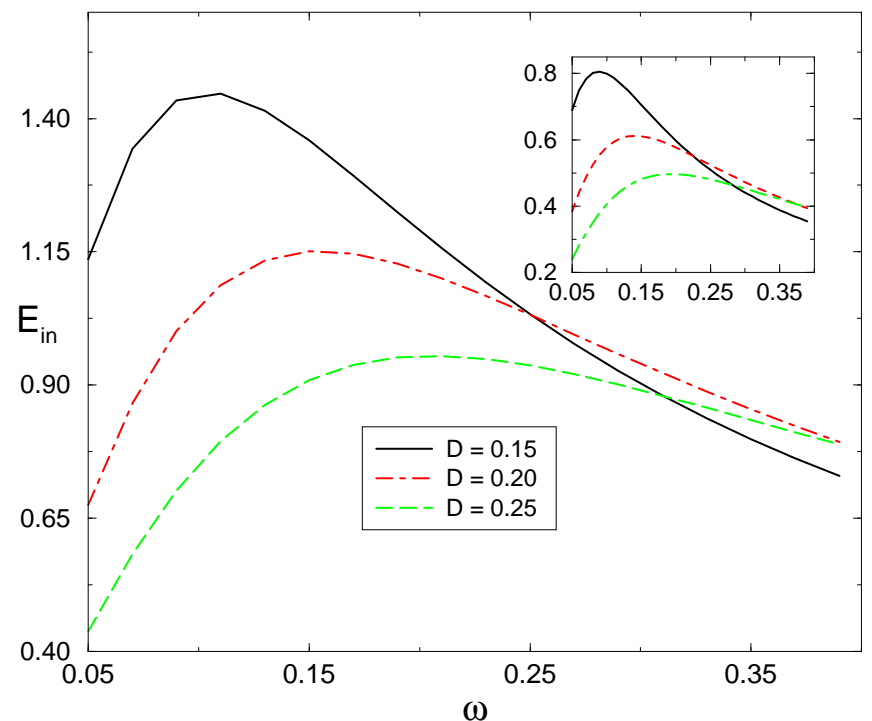

FIG. 2: 


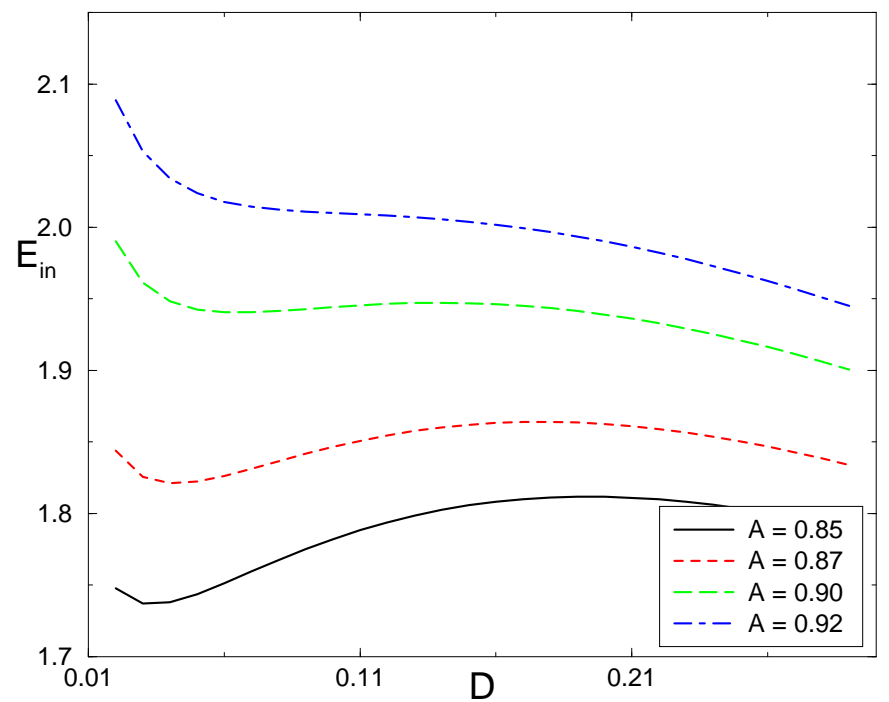

FIG. 3:

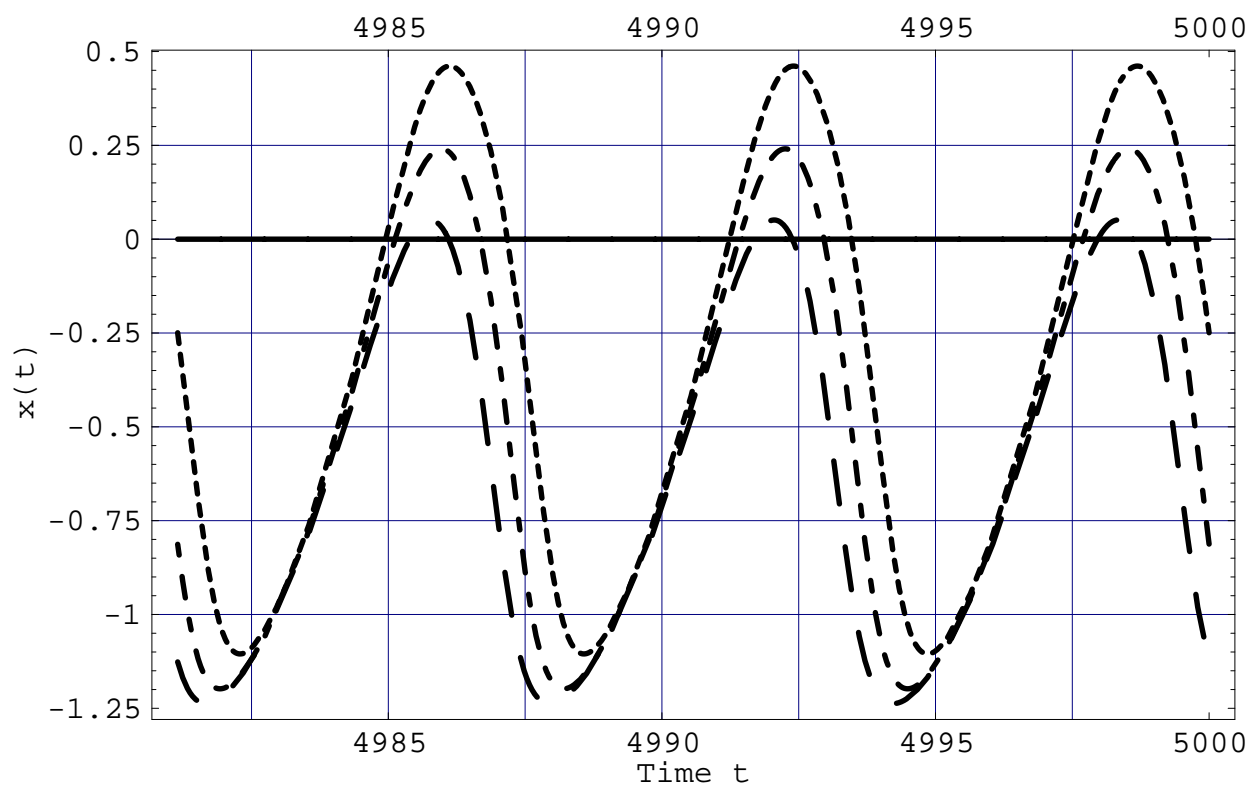

FIG. 4: 